\title{
La modelación con apoyo de software libre y los cambios en los procesos de aprendizaje en matemáticas en los estudiantes de primero de bachillerato
}

\section{Modeling with free software support and changes in the learning processes in mathematics in middle school students}

\section{A modelagem com apoio de software livre e as mudanças nos processos de aprendizagem em matemática em calouros do ensino médio}

\author{
Neli Norma Gonzales Prado* \\ César Augusto Trelles Zambrano** \\ Fecha de recepción: 16 de diciembre de 2015 \\ Fecha de evalucación: 18 de mayo de 2016 \\ Fecha de aceptación: 8 de mayo de 2017 \\ Disponible en línea: 18 de mayo de 2017
}

DOI: http://dx.doi.org/10.18359/reds.1506

Cómo citar este artículo:

Gonzale Prado, N.N. y Trelles, C.A. (2017). La modelación con apoyo de software libre y los cambios en los procesos de aprendizaje en matemáticas en los estudiantes de primero de bachillerato. Revista Educación y Desarollo Social, 11(1), 64-73. DOI: org/10.18359/reds.1506 de Matemáticas y Física en la misma universidad. Correo electrónico: neli.gonzales@ucuenca. edu.ec

** Magíster en Docencia de las Matemáticas, de la Universidad de Cuenca, Ecuador. Profesor de Matemáticas y Física en la misma universidad. Correo electrónico: cesar.trellesz@ucuenca.edu.ec 


\title{
Resumen
}

El presente trabajo muestra los fundamentos principales y algunos aspectos metodológicos en un proyecto de investigación que involucra los procesos de modelación matemática y el uso de recursos tecnológicos en dos instituciones educativas. Las razones principales para llevar a cabo el proyecto han sido la reciente incorporación de la modelación matemática en el currículo ecuatoriano y la escasa investigación a nivel nacional que reporte las ventajas o desventajas de utilizar esta metodología en las aulas. Para la ejecución de la investigación se trabaja mediante la investigación-acción-participación, con un enfoque reflexivo y colaborativo del proceso. Este enfoque cuenta con la participación directa de los involucrados en el proceso de aplicación mediante foros de discusión, mesas redondas y paneles de trabajo.

Palabras clave: modelización matemática, aprendizaje, software.

\begin{abstract}
The present work shows the main fundamentals and some methodological aspects in a research project involving the processes of mathematical modeling and the use of technological resources in two educational institutions. The main reasons to undertake the project have been the recent incorporation of the mathematical modeling in the Ecuadorian curriculum, and the limited research at a national level that reports the advantages or disadvantages of using this methodology in the classroom. For the execution of the study, we worked through action-participation research, with a reflective and collaborative approach to the process. This approach has the direct participation of those involved in the implementation process through discussion forums, roundtables, and work panels.
\end{abstract}

Keywords: Mathematical modeling, learning, software

\section{Resumo}

Este artigo apresenta os principais fundamentos e alguns aspectos metodológicos em um projeto de pesquisa que envolve os processos de modelagem matemática e o uso de recursos tecnológicos em duas instituições de ensino. As principais razões para a realização de o projeto tem sido a recente adição da modelagem matemática no currículo Equatoriano e a escassa pesquisa ao nível nacional que faça o relatório das vantagens ou desvantagens de utilizar esta metodologia em sala de aula. Para a execução da investigação se trabalha por meio de pesquisa-ação-participação, com uma abordagem colaborativa e refletiva do processo. Esta abordagem conta com a participação direta das pessoas envolvidas no processo de implementação através de foros de discussão, mesas redondas e painéis de trabalho.

Palavras-chave: modelagem matemática, aprendizagem, software. 


\section{Introducción}

El Ministerio de Educación del Ecuador, en mayo de 2010, emitió una nueva propuesta curricular para ser aplicada en los niveles de educación general básica y bachillerato general unificado. En ella, matemáticas es una de las áreas de estudio priorizadas por el Ministerio para ejecutar la nueva propuesta, pues en ella se plantea un nuevo enfoque para sus procesos de enseñanza-aprendizaje. En este marco se establece el hecho de que "Adquirir conceptos e instrumentos matemáticos que desarrollen el pensamiento lógico, matemático y crítico para resolver problemas mediante la elaboración de modelos" (Ministerio de Educación (MineDuc), 2015, p. 3) debe constituirse en un eje curricular integrador ${ }^{1}$ de la matemática en el Bachillerato General Unificado (BGU), el cual se apoya en los siguientes ejes del aprendizaje: ${ }^{2}$ abstracción, generalización, conjetura y demostración; integración de conocimientos; comunicación de las ideas matemáticas y el uso de las tecnologías en la solución de problemas, los cuales deben ser trabajados en los diferentes bloques donde se encuentra organizada la disciplina: Números y funciones, Álgebra y Geometría, Matemáticas Discretas y Estadística y Probabilidad.

Idea de mayor grado de generalización del contenido de estudio que articula todo el diseño curricular de cada área, con proyec66 ción interdisciplinaria.

2 Se entienden como el hilo conductor que sirve para articular las destrezas con criterios de desempeño de cada bloque curricular.
En relación con el último eje del aprendizaje, la autoridad considera:

[...] en Matemática muy a menudo hay que realizar cálculos, gráficas, etc. Estas tareas pueden ser ejecutadas mediante software matemático, ahorrando de esta manera valioso tiempo que puede ser empleado en: elaboración de modelos, formulación de hipótesis, de conjeturas, etc. Por tanto el uso racional y eficiente de las tecnologías será una herramienta invaluable en la aplicación de los conocimientos matemáticos para la solución de problemas. (Ministerio de Educación, 2015, p. 5)

Por otro lado, el eje curricular integrador del área en su última parte señala que los estudiantes deben adquirir la capacidad de utilizar la modelación para resolver problemas. De esta manera, el presente trabajo plantea la aplicación de la modelación matemática como estrategia didáctica con apoyo de software educativo, que permita el desarrollo de los aprendizajes de matemáticas en estudiantes de primer año del BGU.

\section{Marco teórico}

En los últimos años la modelación matemática ha resultado atractiva para la comunidad de investigadores en el campo de la educación matemática, pues al ser un tema de investigación relativamente reciente no existe aún una teoría unificada de cómo debe ser trabajada en las aulas. Sin embargo, hasta el momento 
algunos investigadores plantean varias perspectivas como ideas orientadoras, tanto para implementarla en el proceso de enseñanza aprendizaje, como para seguir avanzando en la investigación relacionada con este tema. Kaiser y Sriraman (2006) clasifican estas perspectivas en perspectiva realista, contextual, educativa, sociocrítica, epistemológica y cognitiva. Pero ¿qué es la modelación matemática? Son muchas las aproximaciones conceptuales sobre modelación, por ejemplo, Giordano, Weir y Fox (1997) la definen como "una construcción matemática dirigida a estudiar un sistema o un fenómeno particular del mundo real. Este modelo puede incluir gráficas, símbolos, simulaciones y construcciones experimentales" (p. 34).

Para la obtención de un modelo matemático intervienen muchos factores, como tiempo, conocimiento del contexto y conocimientos matemáticos, entre otros. El proceso de obtención de un modelo matemático a partir de un fenómeno se llama modelización matemática (Villa, 2007). Es importante anotar que las definiciones anteriores hacen referencia a la modelización matemática como actividad científica, en tanto que en el contexto educativo - de manera específica en el aula de clase- se entiende por modelación matemática la actividad, cuya naturaleza se deriva de la actividad científica de modelización matemática y que, más que una herramienta para construir nuevos objetos matemáticos, se convierte en una estrategia que posibilita el entendimiento de un concepto matemático inmerso en un "micromundo" (contexto dotado de relaciones y significados), que prepara al estudiante para ir desarrollando una actitud diferente de preguntarse y abordar los problemas del contexto real (Villa, 2007).

Algunos reportes investigativos señalan que a través de la modelación los estudiantes pueden comprender mejor los contextos donde se desenvuelven; se apoya el aprendizaje de las matemáticas y se promueve el desarrollo de algunas competencias, actitudes y visiones adecuadas hacia las matemáticas (Blum y Borromeo, 2009).

También es preciso indicar que la perspectiva que se categoriza como modelación educativa tiene, como su nombre lo indica, un objetivo claramente pedagógico. Aquí se pueden distinguir dos tipos de corrientes: una didáctica en la que los modelos se utilizan para estructurar y promover el proceso de aprendizaje de los alumnos, y otra que se puede considerar de carácter conceptual, en la cual el papel de la modelación es clave para introducir nuevos conceptos y para desarrollarlos (Trigueros, 2009). Si bien es cierto que existen diferentes perspectivas, todas comparten algunas características, por lo que al elaborar una propuesta que pretenda llevar la modelación como metodología por utilizarse en la clase de matemáticas es difícil ubicarla exclusivamente dentro de una sola. 
En los últimos años en algunos países de América Latina como México, Colombia, Brasil, entre otros, se han desarrollado investigaciones que permiten encontrar resultados acerca de la implementación de la modelación matemática en las aulas.

[... en eneneral, se puede decir que el trabajo en los modelos proporcionó una excelente oportunidad para desarrollar eficazmente los conocimientos de los alumnos, además de ampliar su visión del significado de las matemáticas en la solución de problemas reales. Asimismo, la aplicación de la modelación en la solución ayudó a la mayor parte a sentirse más seguros de sus competencias y valorar de manera diferente la función de los cursos escolares, así como valorar las limitaciones de este tipo de modelación. (Trigueros, 2009, p. 85)

En el caso ecuatoriano, no existe literatura que permita obtener conclusiones acerca de las ventajas o desventajas de la implementación de los procesos de modelación matemática. De igual forma, en la literatura de otros países no se profundiza cómo han influenciado las metodologías de modelación matemáti$\mathrm{ca}$, tanto en los procesos de aprendizaje de los estudiantes, como en su respectivo rendimiento académico.

Los hallazgos presentados respecto a la investigación en modelación matemática no son muy explícitos en cuanto al uso de la tecnología como apoyo a esta metodología, y quienes lo hacen enfatizan en la preparación del profesorado para poder implementarla. El uso de la tecnología en el aula para actividades de modelización matemática puede ser un proceso complejo, no es una simple cuestión de ofrecer computadoras, calculadoras y programas para los alumnos y profesores. Fundamentalmente, se debe tener cuidado de no perder de vista a las matemáticas y el espíritu de la modelización matemática, incluso cuando la tecnología ofrece todas las comodidades y, a veces, hasta algunas de las respuestas (Ang, 2010).

Considerando que la implementación de la modelación matemática persigue objetivos pedagógicos, es importante también no perder de vista algunos aspectos centrales acerca de los procesos de aprendizaje y del rendimiento académico. Al momento de teorizar sobre este tema, Bruner (citado por García, 2012, p. 11) considera que este involucra tres procesos, que son casi simultáneos: la adquisición (que implica información nueva o un refinamiento de la información ya existente), la transformación (que implica manipular el conocimiento para ajustarlo a las nuevas tareas) y la evaluación (para comprobar si la manera como manipulamos la información es la adecuada). Para lograr esto, el proceso educativo debe tener en cuenta la predisposición del individuo hacia el aprendizaje, lo que de una u otra manera implica el carácter emocional con que se asume el aprendizaje en sí mismo. En cuanto rendimiento académico, según Pérez, Ramón y Sánchez (citados por Garbanzo, 2007, p. 46), 
es la suma de diferentes y complejos factores que actúan en la persona que aprende, $y$ ha sido definido como un factor atribuido al logro del estudiante en las tareas académicas; se mide mediante las calificaciones obtenidas, con una valoración cuantitativa, cuyos resultados muestran las materias ganadas o perdidas, la deserción y el grado de éxito académico.

Respecto al uso de las Tecnologías de la Información y Comunicación (TIC) en las aulas, Wengslinky (1998), a través de la prueba National Assessment of Educational Progress (NAEP) en Estados Unidos, concluyó que cuando los profesores estaban lo suficientemente capacitados para dirigir a los estudiantes, el uso de la tecnología parecía estar asociado con mejoras significativas en los logros de aprendizaje en matemáticas.

Hoy en día se habla de sociedad del conocimiento, economía del conocimiento, revolución digital, brecha digital, las TIC. Esta realidad exige, y particularmente a los Gobiernos de los países en vías de desarrollo, un esfuerzo considerable por comprender las determinantes de este fenómeno y poder identificar los mecanismos y las variables por considerar en una estrategia hacia la construcción de la llamada sociedad de la información (Valenti, 2002). En un mundo de permanentes cambios, se considera que para alcanzar la mencionada sociedad del conocimiento es imprescindible que los procesos educativos estén cada vez más acompañados de forma eficiente de recursos tecnológicos.
No obstante lo anterior, algo que no va a cambiar es el poder y la necesidad de interpretar el mundo de manera cuantitativa, así como tampoco la necesidad de convertir información en significados, enfocados a mejorar la calidad de vida, aunque cambien las herramientas específicas, el poder de la cuantificación-matematización continuará. Si realmente queremos que los estudiantes valoren socialmente el conocimiento matemático es necesario arrancar su concepción del nivel utilitario de tal conocimiento y llevarlos al nivel funcional. Tendremos que encontrar los indicadores para que el sistema educativo logre el nivel funcional de la matemática (Cordero, s. f.).

Finalmente, de un análisis bibliográfico efectuado a través de buscadores especializados de la temática, se colige que en nuestro medio no existe información que proporcione evidencias acerca de la relación existente entre el uso de las tecnología aplicada a los procesos de enseñanza-aprendizaje de las matemáticas con el rendimiento académico de los estudiantes, menos aún de aportes que señalen los cambios producidos en los aprendizajes de los estudiantes como consecuencia del uso de la modelación matemática con apoyo de software educativo. Las escasas referencias que se tienen son las tesis de maestría en Docencia de las Matemáticas, de la Universidad de Cuenca, Ecuador, así como de tesis similares en otras universidades que como propuestas de intervención están relacionadas con el uso de recursos tecnológicos para la educación matemática 
y presentan resultados con pequeños grupos de trabajo, como por ejemplo la tesis titulada El aprendizaje de la línea recta y la circunferencia a través de Secuencias didácticas de aprendizaje fundamentadas en la teoría social-cognitivo y desarrollada en geogebra de Barrazueta (2014); o las investigaciones descriptivas, como la realizada por la Universidad Técnica Particular de Loja, referida al uso de TIC en la práctica docente de los maestros de educación básica y bachillerato de la ciudad de Loja. Las investigaciones mencionadas concluyen que el uso inadecuado de recursos tecnológicos por parte de docentes es explicado en parte por la carencia de capacitación formal, e incluso el rechazo y la oposición al uso de las TIC en centros públicos (Valdivieso, 2010).

\section{Características de la modelación matemática}

De acuerdo con las investigaciones realizadas, la modelación matemática deberá cumplir con las siguientes características.

Las actividades de modelación tienen que ser seleccionadas o adaptadas para que los estudiantes las tomen como "realistas", de tal manera que respondan a una situación de su entorno y realidad y los ubiquen en una situación de desequilibrio conceptual.

70 Los maestros deben ser las personas más capaces de interpretar el significado de los trabajos de modelación de sus estudiantes, y así tener un listado de su forma de pensar, reflexionar y mediar entre la validez matemática de su respuesta y su reflexión.

En esta estrategia es muy importante la interpretación para poder operar/ actuar matemáticamente en el mundo, con especificaciones claras para juzgar una solución.

\section{Metodología}

Se eligen actividades seleccionadas, como por ejemplo la actividad "El problema del trabajo de vacaciones" (ver figura 1 y tabla 1) o se construyen nuevas actividades junto con los docentes de las instituciones de implementación, para ubicarlas dentro del currículo exigido por el Ministerio de Educación. Asimismo, se planifica y se prepara muy detenidamente la implementación de cada actividad, adaptándola y precisando expectativas acerca del trabajo de los estudiantes. También se evalúa la ejecución de las diferentes actividades y se estudia la percepción de los estudiantes al trabajar con actividades de este tipo.

Por la factibilidad de espacio físico y acceso del equipo investigador, se aplicará la propuesta en dos instituciones educativas de la provincia del Azuay. Para la ejecución de la propuesta se trabajará mediante la investigación-acción-participación, con un enfoque reflexivo y colaborativo del proceso, enfoque que contará con la participación directa de los involucrados en el proceso de 
aplicación mediante foros de discusión, mesas redondas, paneles de trabajo, etc.

\section{Conclusiones}

\section{El abordaje de la modelación matemática como estrategia metodológica en las aulas de clase se vuelve primordial para la educación matemática en el ámbito ecuatoriano. Al ser un tema reciente- mente incorporado en el currículo, es de}

vital importancia aportar a la literatura con datos que sustenten si el hecho de incorporarla en las aulas es beneficioso para el proceso educativo.

Existen algunos hallazgos interesantes referentes al tema, como por ejemplo las reacciones generadas en los estudiantes, así como el nivel de preparación del profesorado para poder liderar en el aula actividades de este tipo.

En las vacaciones pasadas, Carla inició un negocio de ventas en el parque central del lugar. Sus vendedores llevaron dulces, empanadas y bebidas por todos los lados del parque.

El negocio tuvo un éxito tremendo. El próximo año, Carla tiene expectativas de que todos sus vendedores querrán trabajar nuevamente con ella. Pero desde la administración municipal le han comunicado que no se permitirán tantos vendedores a futuro. Entonces, Carla necesita la ayuda de ustedes, para decir qué vendedores contratar. Solamente va a poder seleccionar un tercio de ellos a tiempo completo, otro tercio de medio tiempo y el resto no podrá ser contratado.

La siguiente tabla presenta una muestra del desempeño de 9 vendedores que participaron en las vacaciones pasadas. Para hacer esta tabla, Carla revisó su registro de trabajo, sumó el número de horas que trabajaron y la cantidad de dinero que ingresó al negocio cada mes, además, clasificó la afluencia de gente en alto, normal y bajo (ver tabla 1). Carla quiere contratar a las personas que le van a generar mayores ingresos, pero no sabe cómo compararlos, pues trabajaron distintos números de horas y bajo distintas condiciones de afluencia.

La tarea de ustedes es proponer un procedimiento equitativo para decidir a quiénes contratar.

Escriban una carta a Carla, diciendo cómo puede evaluar a todos los vendedores y cómo decidir a quiénes elegir para trabajar, tanto a tiempo completo, como a medio tiempo. Expliquen cómo funciona su procedimiento para las nueve personas de la tabla. Muestren detalles para que Carla pueda averiguar sus resultados y expliquen su razonamiento para que pueda decidir si el método propuesto por ustedes es adecuado para los objetivos del negocio.

Figura 1. Contextualización del problema del trabajo de vacaciones

Fuente: elaboración propia a partir de Lesh y Lehrer (2000). 
Tabla 1. Datos de la contextualización del problema del trabajo de vacaciones

\begin{tabular}{|c|c|c|c|c|c|c|c|c|c|}
\hline \multicolumn{10}{|c|}{ Horas de trabajo } \\
\hline & \multicolumn{3}{|c|}{ Junio } & \multicolumn{3}{|c|}{ Julio } & \multicolumn{3}{|c|}{ Agosto } \\
\hline & Alto & Normal & Bajo & Alto & Normal & Bajo & Alto & Normal & Bajo \\
\hline Daniela & 12,5 & 15 & 9 & 10 & 14 & 17,5 & 12,5 & 33,4 & 35 \\
\hline Ximena & 5,5 & 22 & 15,5 & 53,5 & 40 & 15,5 & 50 & 14 & 23,5 \\
\hline Carlos & 12 & 17 & 14,5 & 20 & 25 & 21,5 & 19,5 & 20,5 & 24,5 \\
\hline Sofía & 19,5 & 30,5 & 34 & 20 & 31 & 14 & 22 & 19,5 & 36 \\
\hline Víctor & 19,5 & 26 & 0 & 36 & 15,5 & 27 & 30 & 24 & 4,5 \\
\hline Ana & 13 & 4,5 & 12 & 33,5 & 37,5 & 6,5 & 16 & 24 & 16,5 \\
\hline Edison & 26,5 & 43,5 & 27 & 67 & 26 & 3 & 41,5 & 58 & 5,5 \\
\hline Paola & 7,5 & 16 & 25 & 16 & 45,5 & 51 & 7,5 & 42 & 84 \\
\hline Miguel & 0 & 3 & 4,5 & 38 & 17,5 & 39 & 37 & 22 & 12 \\
\hline \multicolumn{10}{|c|}{ Ingreso de dinero (en dólares USD) } \\
\hline & \multicolumn{3}{|c|}{ Junio } & \multicolumn{3}{|c|}{ Julio } & \multicolumn{3}{|c|}{ Agosto } \\
\hline & Alto & Normal & Bajo & Alto & Normal & Bajo & Alto & Normal & Bajo \\
\hline Daniela & 690 & 780 & 452 & 699 & 758 & 835 & 788 & 1732 & 1462 \\
\hline Ximena & 474 & 874 & 406 & 4612 & 2032 & 477 & 4500 & 834 & 712 \\
\hline Carlos & 1047 & 667 & 284 & 1389 & 804 & 450 & 1062 & 806 & 491 \\
\hline Sofía & 1263 & 1188 & 765 & 1584 & 1668 & 449 & 1822 & 1276 & 1358 \\
\hline Víctor & 1264 & 1172 & 0 & 2477 & 681 & 548 & 1923 & 1130 & 89 \\
\hline Ana & 1115 & 278 & 574 & 2972 & 2399 & 231 & 1322 & 1594 & 577 \\
\hline Edison & 2253 & 1702 & 610 & 4470 & 993 & 75 & 2754 & 2327 & 87 \\
\hline Paola & 550 & 903 & 928 & 1296 & 2360 & 2610 & 615 & 2184 & 2518 \\
\hline Miguel & 0 & 125 & 64 & 3073 & 767 & 768 & 3005 & 1253 & 253 \\
\hline
\end{tabular}

Fuente: elaboración propia a partir de Lesh y Lehrer (2000).

\section{Referencias}

Ang, K. C. (2010). Teaching and Learning Mathematical Modelling with Technology. En W.-C. Yang, M. Majexski, $72 \quad$ A. Tilaky W. Ping (Eds.), Fifteenth Asian Technology Conference in Mathematics. Kuala-Lumpur: Mathematics and Technology. Recuperado de http:// atcm.mathandtech.org/ep2010/ invited/3052010_18134.pdf

Barrazueta, J. (2014). El aprendizaje de la línea recta y la circunferencia a través de secuencias didácticas de aprendizaje fundamentadas en la teoría social-cognitiva y desarrollada en geogebra. Cuenca: Universidad de Cuenca. 
Blum, W. y Borromeo, R. (2009). Mathematical Modelling: Can It Be Taught And Learnt? Journal of Mathematical Modelling and Application, 1(1), 45-58.

Cordero, F. (s. f.). La modelación y la enseñanza de las matemáticas. Seminario Repensar las Matemáticas. Recuperado de https://repensarlasmatematicas. files.wordpress.com/2012/09/44artvideoconf-08-2012.pdf

Garbanzo, G. (2007). Factores asociados al rendimiento académico en estudiantes universitarios, una reflexión desde la calidad de la educación superior pública. Educación, 31(1), 43-63.

García, J. (2012). La educación emocional, su importancia en el proceso de aprendizaje. Educación, 36(1), 1-24.

Giordano, F., Weir, M. y Fox, W. (1997). A first Course in Mathematical Modeling (2da ed.). Nueva York: Brooks/Cole Publishing Company

Kaiser, G. y Sriraman, B. (2006). A global survey of international perspectives on modelling in mathematics education. ZDM, 38(3), 302-310.

Lesh, R. y Lehrer, R. (2000). Iterative refinement cycles for videotape analyses of conceptual change. En A. Kelly y R. Lesh (Eds.), Handbook of Research Design in Mathematics and Science Education (pp. 665-708). Mahwah: Lawrence Erlbaum Associates.
Ministerio de Educación. (2 de Septiembre de 2015). Lineamientos Curriculares para el Bachillerato General Unificado. Quito: autor.

Trigueros, M. (2009). El uso de la modelación en la enseñanza de las matemáticas. Innovación Educativa, 9(46), 75-87.

Valdivieso, T. (2010). Uso de TIC en la práctica docente de los maestros de educación básica y bachillerato de la ciudad de Loja. Revista Electrónica de Tecnología Educativa, 33, 1-13.

Valenti, P. (2002). La sociedad de la información en América Latina y el Caribe: TIC y un nuevo marco institucional. Revista Iberoamericana de Ciencia, Tecnología, Sociedad e Innovación, (2).

Villa, J. (2007). La modelación como proceso en el aula de Matemáticas. Un marco de referencia y un ejemplo. Tecno-Lógicas, 19, 63-85.

Villa, J. (octubre de 2009). Presente y futuro de la investigación en modelación en educación matemática en Colombia. Uninades. Recuperado de http://funes. uniandes.edu.co/756/1/presente.pdf

Wengslinky, H. (1998). Does It Compute? The Relationship Between Educational Technology and Student Achievement in Mathematics. Recuperado de http:// files.eric.ed.gov/fulltext/ED425191.pdf 